\section{Aortogastric fistula secondary to pancreatitis}

\author{
A Ardolino \\ S G Appleton
}

J R Soc Med 2007;100:239-240

\section{INTRODUCTION}

Primary aortogastric fistulas are rare and usually associated with aortic aneurysms. We report a primary aortogastric fistula of unusual aetiology.

\section{CASE HISTORY}

A 68-year-old woman was admitted with acute pancreatitis (Glasgow Score two). She was treated with intravenous fluids and antibiotics. Abdominal CT showed small bilateral pleural effusions and a large hiatus hernia but was otherwise unremarkable. Her recovery was uneventful and she was discharged after twelve days.

She was readmitted two weeks later with breathlessness and new onset atrial fibrillation. At this point she had mild epigastric pain and raised inflammatory markers. A repeat abdominal CT showed a fluid collection adjacent to the hiatus hernia in the posterior mediastinum (Figure 1). Total parenteral nutrition was commenced and the plan was to drain the collection.

A few days later she developed severe epigastric pain followed by episodes of melaena, haematemesis and hypotension. She remained hypotensive despite vigorous fluid resuscitation and blood transfusion. Urgent upper gastrointestinal endoscopy was performed. The view was poor due to fresh blood and clots in the oesophagus and it was not possible to enter the stomach.

At emergency laparotomy two litres of infected fluid was aspirated from a pancreatic pseudocyst. Two-thirds of the stomach had herniated into the chest and was irreducible. Gastrostomy revealed fresh blood and clots in the intrathoracic stomach. An arterial bleeding point was identified within the stomach overlying the thoracic aorta. Attempts to stop the bleeding were unsuccessful and the patient died. A post mortem showed a fistula between the thoracic aorta and the intrathoracic stomach.

\section{DISCUSSION}

Aortoenteric fistulas are uncommon and are frequently fatal. Most are secondary fistulas occurring after aortic replacement. Primary aortoenteric fistulas are very rare and

Correspondence to: A Ardolino

E-mail: tonia@entee.co.uk

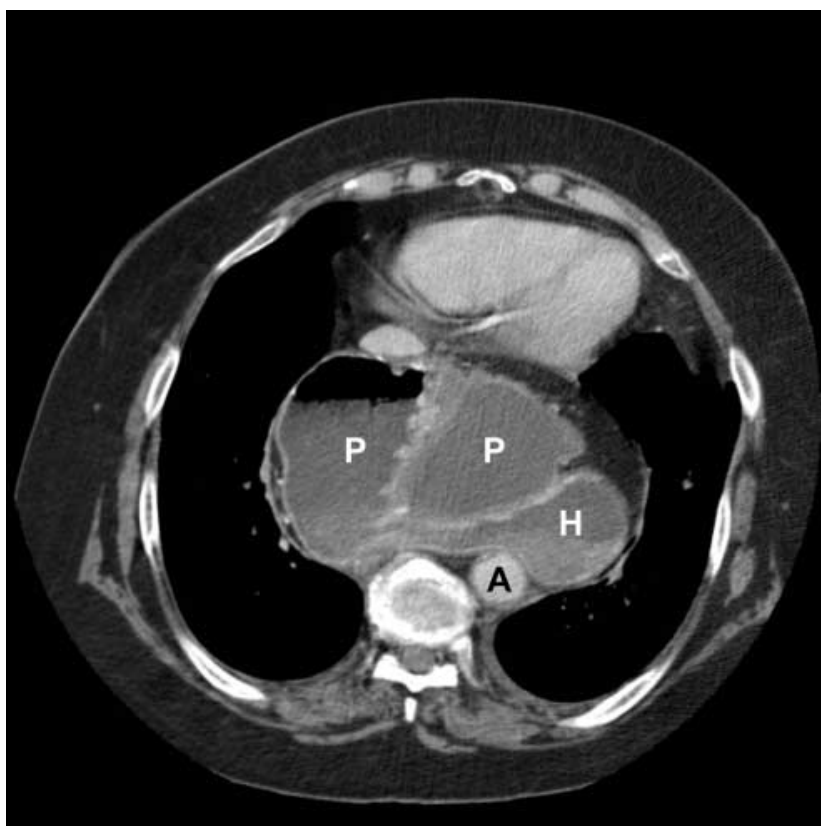

Figure 1 CT scan showing the close relatinship of the thoracic aorta $[A]$, pancreatic pseudocyst $[\mathrm{P}]$ and hiatus hernia $[\mathrm{H}]$

are due to spontaneous erosion of the aorta into the gastrointestinal tract. The incidence of primary aortoenteric fistulas is reported at about $0.07 \%$ of the general population. The male to female ratio is $3: 1$ and the mean age of affected people is 64 years. ${ }^{1}$

A recent review revealed that $83 \%$ of patients with a primary aortoenteric fistula have an associated aortic aneurysm. ${ }^{1}$ Other reported causes include infection, tumour, radiotherapy, pancreatic cancer and peptic ulcer disease. $^{2}$

Primary aortoenteric fistulas can affect any part of the gastrointestinal tract. They are most common in the third or fourth part of the duodenum, with fistulae in these regions accounting for $54 \%$ of all cases. ${ }^{1-3}$ Aortogastric fistulas account for $2 \%$ of fistulas.

Upper gastrointestinal bleeding is the most common presentation of aortoenteric fistulas. Melaena occurs in 40 $55 \%$ or cases and haematemesis in $64 \% .^{4}$ There is often a 'herald bleed' followed by massive exsanguination. In $11 \%$ of cases there is a triad of abdominal pain, gastrointestinal haemorrhage and a palpable abdominal mass. ${ }^{1}$ In others the main symptom is fever. ${ }^{4}$

In a stable patient with an aortoenteric fistula it may be possible to use a covered aortic stent graft to obtain haemostasis. Endovascular repair has an increasing role in the treatment of aortic disease; it is minimally invasive and useful for patients with other morbid factors. Stent graft implantation has successfully been used to treat aortic aneurysms, dissections, to control bleeding from aortoureteric fistulas and in aortic trauma. ${ }^{5,6}$ 
Pancreatic pseudocysts are a recognized complication of pancreatitis. They commonly occur after two weeks. A pseudocyst is a collection of pancreatic enzymes, inflammatory exudate and necrotic debris. It is thought that a combination of fat necrosis and the spillage of pancreatic enzymes into the mediastimun contributed to the fistula formation in this patient.

This is a remarkable case due to the rare location of the fistula and the lack of an associated aortic aneurysm. A search of the English literature reveals it is the first report of an aortogastric fistula secondary to pancreatitis.

Competing interests None declared.

Funding There was no funding or sponsorship for this report.

\section{REFERENCES}

1 Saers SJF, Scheltinga MRM. Primary aortoenteric fistula. Br J Surg 2005;92:143-52

2 Farber A, Grigoryants V, Palac DM, Chapman T, Cronenwett JL, Powell RJ. Primary aortoduodenal fistula in a patient with a history of intravesical therapy for bladder cancer with bacillus Calmette-Guerin: Review of primary aortoduodenal fistula without abdominal aortic aneurysm. J Vasc Surg 2001;33:868-73

3 Lee JT, Saroyan M, Belzberg G, Pianim NA, Bongard FS. Primary aortoenteric fistula: Computed tomographic diagnosis of an atypical presentation. Ann Vasc Surg 2001;15:251-54

4 Van Olffen TBM, Knippenberg LHA, Van der Vliet JA, Lastdrager WB. Primary aortoenteric fistula: report of six new cases. Cardiovasc Surg 2002;10:551-54

5 Rousseau H, Bolduc JP, Dambrin C, Marcheix B, Canevet G, Otal P. Stent-graft repair of thoracic aortic aneurysms. Tech Vasc Interv Radiol 2005:8:61-72

6 Ota T, Tsuji Y, Kawasaki R, Taniguchi T, Morimoto Y, Okita Y. Endovascular treatment of aortoureteric fistula. J Endovasc Ther 2005:12:411-13

\section{WIN THE PUBLICATION GAME}

Kamran Abbasi and the Young Fellows' Committee of the Royal Society of Medicine invite you to learn how to improve your chances of getting published and advancing your career in one day...

\section{'The most useful day I have had in years' Lalitha D'Souza, Delegate, 3 June 2006}

Run by Kamran Abbasi, Editor of the Journal of the Royal Society of Medicine, this is an extremely useful day for any trainee. Described as 'a triumph' by The Lancet (15 April 2006), Kamran's course is the ideal guide to lead you through the world of medical publishing.

For further information or to book online, visit the diary page of the Young Fellows' Committee on the RSM website at www.rsm.ac.uk/yf/yfmeet.php Alternatively you can contact Anna Jesse: tel (+44) 0207290 3846; fax (+44) 0207290 2989; email young.fellows@rsm.ac.uk 